\title{
Schwarzschild Quantum Light Geodesics Metric: A Pair of BH-Inner WH
}

\author{
Jean Perron (i) \\ Department of Applied Sciences, Université du Québec à Chicoutimi, Chicoutimi, Canada \\ Email: jean_perron@uqac.ca
}

How to cite this paper: Perron, J. (2021) Schwarzschild Quantum Light Geodesics Metric: A Pair of BH-Inner WH. Journal of High Energy Physics, Gravitation and Cosmology, 7, 1089-1101. https://doi.org/10.4236/jhepgc.2021.73064

Received: June 1, 2021

Accepted: July 5, 2021

Published: July 8, 2021

Copyright (c) 2021 by author(s) and Scientific Research Publishing Inc. This work is licensed under the Creative Commons Attribution International License (CC BY 4.0).

http://creativecommons.org/licenses/by/4.0/

(c) (i) Open Access

\begin{abstract}
In this article we hypothesized that the arrow of time and space evolve in a discontinuous way in the form of quanta $\left(t=n t_{P}, s=m l_{P}\right)$. We applied this reasoning to the light geodesics of Schwarzschild's metric $\left(\mathrm{d} s_{\min }=l_{P}\right)$ and obtained different characteristics of the $\mathrm{BH}$. Indeed, quantum light geodesics show that inside the $\mathrm{BH}$ a WH is formed and the mass (energy) is not directing towards the singularity $r \rightarrow 0$ but rather around the $\mathrm{BH}$ near the $\mathrm{EH}$ as a thick skin (tickness $\frac{R_{s}}{2}$ ). The total relativistic energy invariant is satisfied for the entire route of the photons. Subsequently, as mass (energy) is found directly at the $\mathrm{EH}$, we applied the quantum tunnel effect in simple semiclassical analysis, and we obtained that particles like protons can leave the $\mathrm{EH}$ and that the energy associated with them is in the order of magnitude of Hawking's radiation. However, the energy of the protons is not necessarily identified with that of the black body (photonic or electromagnetic). Finally, it would be interesting to see the impacts of this quantum light geodesics $\left(\mathrm{d} s_{\min }=l_{P}\right)$ on other cases like Kerr's metric.
\end{abstract}

\section{Keywords}

Black Hole, White Hole, Schwarzschild Metric, Quantum Spacetime, Tunnel Effect

\section{Introduction: Formulation of the Model Initial Concept}

The idea behind this article comes from a rather long reflection about the manufacture of the universe as well as a model of cosmology already published [1]. The prediction of the possible existence of black hole (BH) is not recent (John Michell in 1783, Pierre Simon de Laplace in 1796). Their properties, although often relatively abstract and difficult to grasp, are fascinating. Since then, a large 
number of physicists and mathematicians have continued to develop and understand what the characteristics of these strange objects might be complex and indirectly observed only once [2]. It was the general relativity (GR) theory as well as the first development of Schwarzschild that brought to light the main characteristics of these objects [3]. Subsequently, other developments and understanding were added to that of Schwarzschild, BH in rotation [4], charged electrically [5]. A large number of publications present the various theories relating to $\mathrm{BH}$, we refer the reader to the thesis of Bardoux [6] which presents a good review of the $\mathrm{BH}$ metrics.

One of the fundamental problems related to the models of BH lies in the mathematical expression within them, which predicts that the energy within the event horizon (EH) invariably follows its movement towards a central singularity $r \rightarrow 0$. Although mathematical, this concentration of energy tending towards infinity to this singularity poses a problem or at least a plot. It is the infinite curvature of space, predicted by the metric of the GR that leads to this state of infinite concentration of energy.

The model proposed here (a quantum light geodesic metric) is based on the idea of quantifying two fundamental variables of the universe: space (the arrow of space increases confirmed by the expansion of the universe) and time (the arrow of time increases confirmed by observation) as proposed by the spacetime of the GR. The modern quantization of our understanding of the universe is approximately 120 years old from Planck (1900). Much effort has been made in the equation of quantum effects related to spacetime [7]. Closer to us, the theory of loop quantum gravity LQG develops a detailed understanding of this quantization of spacetime with the notions of loop, spin-network, foam [8]. In this article, we borrow a similar idea of the quantization of spacetime (quanta). Indeed, we accept that the notion of infinitesimal, when used in physical calculation in the context of algebraic or differential equations, imposes limit values as follows:

$$
\mathrm{d} s_{\min } \geq l_{P}=\sqrt{\frac{h G}{2 \pi c^{3}}} \text { and } \mathrm{d} t_{\min } \geq t_{P}=\sqrt{\frac{h G}{2 \pi c^{5}}}
$$

This is the idea of quantas of space and time. As an example, the derivative that could be called quantum for this function would be:

$$
\begin{aligned}
& f(t)=a t^{2} \\
& \frac{\mathrm{d} f(t)}{\mathrm{d} t}=\lim _{q \rightarrow t_{P}} \frac{f\left(t+t_{P}\right)-f(t)}{t_{P}}=\frac{a\left(t+t_{P}\right)^{2}-a t^{2}}{t_{P}}=\frac{a\left(t^{2}+2 t t_{P}+t_{P}^{2}\right)-a t^{2}}{t_{P}} \\
& \frac{\mathrm{d} f(t)}{\mathrm{d} t}=2 a t+a t_{P}
\end{aligned}
$$

It is clear that at the macroscopic scale, the correction $a t_{P} \sim 10^{-43} a$ is negligible but for a very high value of $a$, as observed in cosmology or astrophysics (e.g. combination of Planck's scale variables or high energy phenomenon), it is possible to obtain not insignificant quantum effect (see a state equation, Perron, 2021) [1]. For example, we also find: 


$$
\begin{gathered}
f(t)=\sin t, \quad \frac{\mathrm{d} f(t)}{\mathrm{d} t_{q}}=\cos \left(t+\frac{t_{P}}{2}\right) \\
f(t)=\frac{1}{t}, \quad \frac{\mathrm{d} f(t)}{\mathrm{d} t_{q}}=\frac{-1}{t^{2}+t t_{P}}
\end{gathered}
$$

For example, at the particle acceleration scale, if we consider the exchange of energy, we can estimate that the variation in total energy associated with the acceleration of the particle can be expressed as:

$$
\begin{gathered}
P=\frac{\mathrm{d} E_{t}}{\mathrm{~d} t}=\frac{\mathrm{d} E_{t}}{\mathrm{~d} v} \frac{\mathrm{d} v}{\mathrm{~d} t} \\
P_{q}=\frac{\mathrm{d} E_{t}}{\mathrm{~d} t}=\frac{\mathrm{d} E_{t}}{\mathrm{~d} v} \frac{\mathrm{d} v}{t_{P}} \\
\Delta_{q}=\frac{\frac{\mathrm{d} E_{t}}{\frac{\mathrm{d} t}{\mathrm{~d} E_{t}}}}{\mathrm{~d} t_{q}}=\frac{\frac{\mathrm{d} v}{\frac{\mathrm{d} t}{t_{P}}}=\frac{t_{P}}{\mathrm{~d} t} \sim \frac{10^{-43}}{10^{-15}} \sim 10^{-28}}{}
\end{gathered}
$$

Thus for phenomena of energy exchange in the order of the femtosecond $\left(10^{-15}\right.$ s), the quantum part of the experiment is worth $\sim 10^{-28}$ of the total which is still undetectable with today's instruments ([9] atomic clock precision $\Delta t \sim 10^{-19} \mathrm{~s}$ ). In general, although it may seem strange, if we accept the quantification of fundamental variables such as time and space, in the field of physics (quantum scale), the notion of infinitesimal tends to zero $(\Delta t \rightarrow 0)$ associated with space and time does not exist. This idea of the non-existence of $(\Delta x \rightarrow 0)$ is compatible with Heisenberg's uncertainty principle because it also delimits the notion of strict infinity $\infty$ that does not exist in physics either.

\section{Schwarzschild Quantum Light Geodesic Metric (SCK)}

The idea of quantum Schwarzschild metric KSC is not new [10] [11]. However, the way in which this quantization is done may differ depending on our understanding of quantification. For example, we can admit a small quantum variation of $r$ proposed by Kazakov and get interesting results about the internal structure of the $\mathrm{BH}$ according to the choice of a quantum variation parameter $a$, which affect $r$. In this article, if we look at the energy part of the evolution of a physical phenomenon, we postulate that spacetime has the quantum characteristic even for light. In admitting this hypothesis of quantum spacetime, we must consider that the light geodesics, normally accepted as zero length in theory ( $\mathrm{d} s \equiv 0$ or proper time $\tau \equiv 0$ ) are also quanta. Thus, for a Schwarzschild metric (spherical symmetry in the void), we find a priori,

$$
\begin{aligned}
& c^{2} \mathrm{~d} \tau^{2}=\mathrm{d} s^{2}=l_{P}^{2}=\frac{h G}{2 \pi c^{3}} \\
& \leq-\left(1-\frac{R_{s}}{r}\right) c^{2} \mathrm{~d} t^{2}+\left(1-\frac{R_{s}}{r}\right)^{-1} \mathrm{~d} r^{2}+r^{2}\left(\mathrm{~d} \theta^{2}+\sin ^{2} \theta \mathrm{d} \varphi^{2}\right)
\end{aligned}
$$

With the radius of Schwarzschild $R_{s}=\frac{2 G M}{c^{2}}$, 
It should be noted here that this metric does not meet Minkowski's metric (flat) $r \rightarrow \infty$. An amendment is required for this condition. This SCK light geodesic metric satisfies Minkowski.

$$
l_{P}^{2} \leq-\left(1-\frac{R_{s}}{r}\right) c^{2} \mathrm{~d} t^{2}+2\left(1-\frac{R_{s}}{r}\right)^{-1} \mathrm{~d} r^{2}+r^{2}\left(\mathrm{~d} \theta^{2}+\sin ^{2} \theta \mathrm{d} \varphi^{2}\right)
$$

Let's do the developments to find the quantum light geodesics and other conclusions. For radial light geodesics, $(\mathrm{d} \theta=\mathrm{d} \varphi=0)$ we find:

$$
\frac{l_{P}^{2}}{\mathrm{~d} t^{2}}=-\left(1-\frac{R_{s}}{r}\right) c^{2}+2\left(1-\frac{R_{s}}{r}\right)^{-1} \frac{\mathrm{d} r^{2}}{\mathrm{~d} t^{2}}
$$

Based on our hypothesis of the quantization of spacetime, the element associated with $l_{P}$ the $\mathrm{d} t$ variable is considered the value of $t_{P}$ (quanta), then,

$$
\frac{l_{P}^{2}}{t_{P}^{2}}=c^{2}=-\left(1-\frac{R_{s}}{r}\right) c^{2}+2\left(1-\frac{R_{s}}{r}\right)^{-1} \frac{\mathrm{d} r^{2}}{\mathrm{~d} t^{2}}
$$

Isolate for the speed of photons, we find a remarkable equation:

$$
\frac{\mathrm{d} r^{2}}{\mathrm{~d} t^{2}}=\frac{1}{2}\left(1-\frac{R_{s}}{r}\right) c^{2}+\frac{1}{2}\left(1-\frac{R_{s}}{r}\right)^{2} c^{2}
$$

either,

$$
\frac{\mathrm{d} r}{\mathrm{~d} t}= \pm c \sqrt{\frac{1}{2}\left(1-\frac{R_{s}}{r}\right)+\frac{1}{2}\left(1-\frac{R_{s}}{r}\right)^{2}}
$$

For $r \rightarrow \infty$, we find Minkowski (flat),

$$
\frac{\mathrm{d} r}{\mathrm{~d} t}= \pm c
$$

And for $r \rightarrow 2 l_{P} \quad\left(r\right.$ minimal for this metric is $\left.R_{s}^{\min }=2 l_{P}\right)$, we find:

$$
\frac{\mathrm{d} r}{\mathrm{~d} t} \rightarrow \pm \infty
$$

The equations of quantum light geodesics $\left(\mathrm{d} s=l_{P}\right)$ are of the form.

$$
c t_{q}= \pm \int \frac{\sqrt{2} \mathrm{~d} r}{\sqrt{\frac{R_{s}^{2}}{r^{2}}-\frac{3 R_{s}}{r}+2}}
$$

either,

$$
c t_{q}= \pm \frac{\sqrt{2}}{2} \sqrt{R_{s}^{2}-3 R_{s} r+2 r^{2}} \pm \frac{3}{4} \ln \left[2 \sqrt{2} \sqrt{R_{s}^{2}-3 R_{s} r+2 r^{2}}+4 r-3\right]+C
$$

Figure 1 shows the photons quantum light geodesics according to the $+\mathrm{d} r / \mathrm{d} t$ (leaving photons) of the conventional Schwarzschild metric and the quantum one. First, we find that the quantum light geodesics are well defined for $r \geq R_{s}$. Second, the quantum light geodesics are not defined for $\frac{R_{s}}{2}<r<R_{s}$ because the value of the radical is negative and the logarithm argument is negative. Third, for the value of $r<\frac{R_{s}}{2}$ the values of geodesics are partially defined 


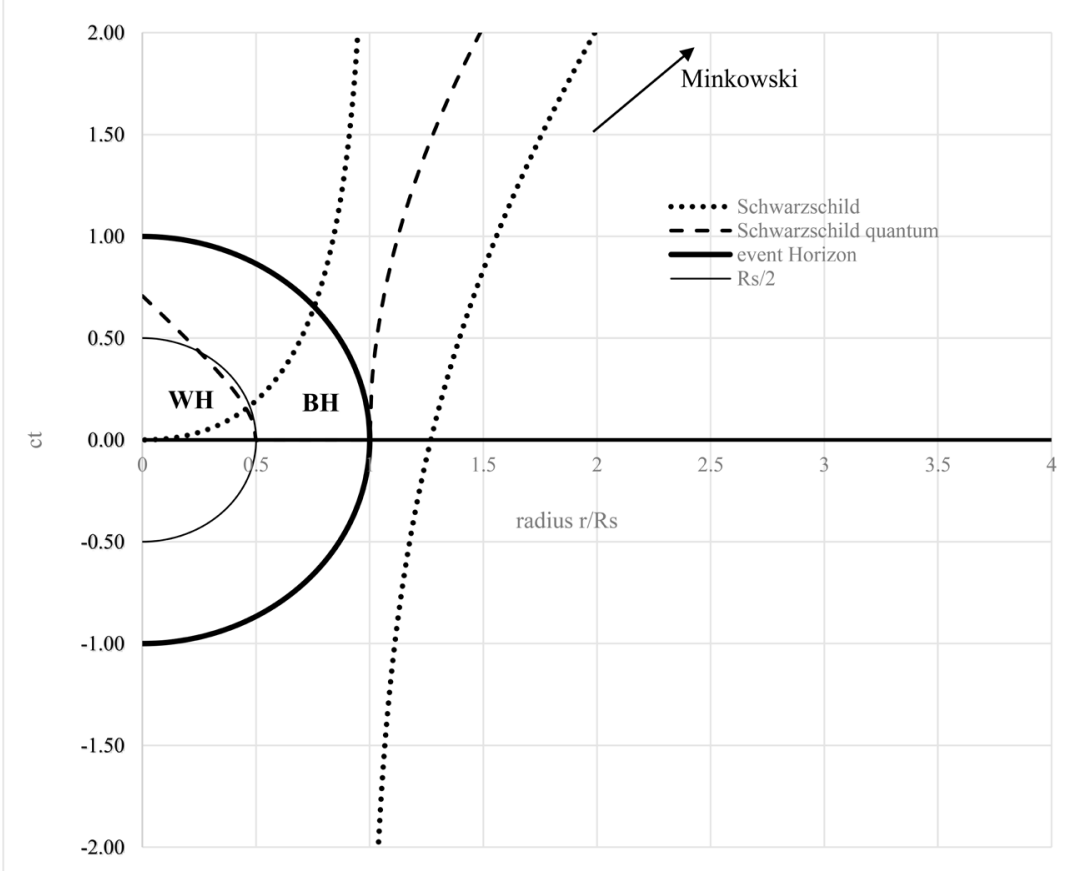

Figure 1. Comparison between two Schwarzschild metrics (conventional and quantum light geodesic).

(the radical is positive but the argument of the logarithm is negative). Finally, we see that for the $\mathrm{BH}$ zone $\frac{R_{s}}{2}<r<R_{s}$ the spacetime is not defined precisely as usual. At $r=R_{s}$ the variation is less rapid. Then, for the quantum case, the photons cross the EH more slowly and remain in this zone between $\frac{R_{s}}{2}<r<R_{s}$. The mass (energy) is stacked "layer per layer" from the outside during the growth of the BH. The geodesics are vertical for $r=R_{s}$ and $\frac{R_{s}}{2}$. However, the photons are no longer rotating as strongly on the EH. This element could be a characteristic to validate this metric.

Figure 2 shows the photons speed. We have a particular zone $\frac{R_{s}}{2}<r<R_{s}$ for which the speed is undetermined (imaginary) but on both sides, the speed $V$ is worth 0 . This zone is like the elongated extension of the point $r=R_{s}$. This zone included $\frac{R_{s}}{2}<r<R_{s}$ could accumulate energy because we observe the speed is positive at $r<\frac{R_{s}}{2}$ a theoretically empty zone of energy that has the characteristics of a white hole WH ([12] a central spherical zone where matter (energy) is ejected outside towards the edge of the $\mathrm{BH}$ zone). This notion of $\mathrm{WH}$ within the $\mathrm{BH}$ is special because it allows to contain the $\mathrm{WH}$ in the $\mathrm{BH}$ and avoid the explosion of the $\mathrm{WH}$, an idea already envisaged [13]. However, what is special here is the existence of a $\mathrm{WH}$ within a $\mathrm{BH}$. 


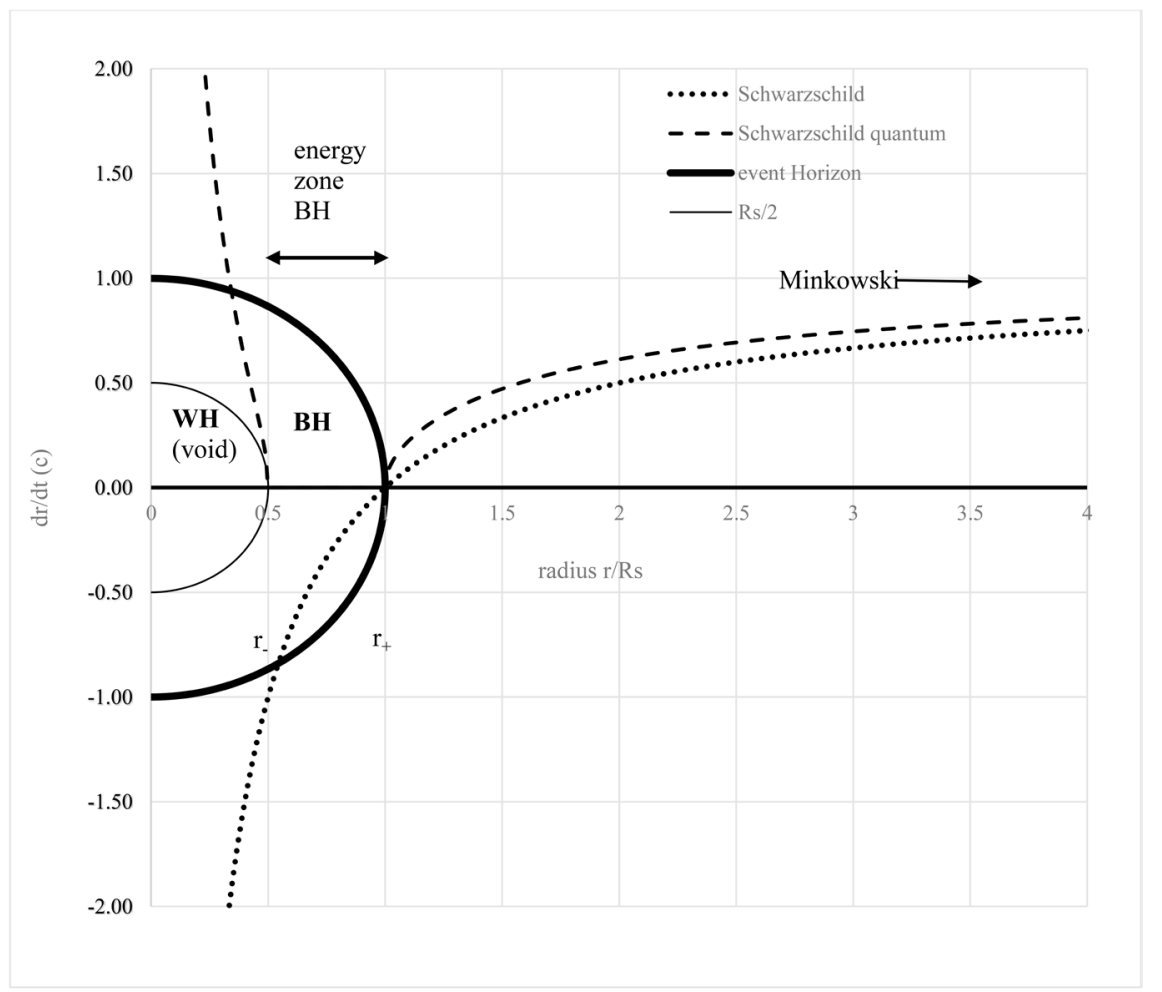

Figure 2. Photon speed, Schwarzschild typical and quantum luminous geodesics.

This inner zone $\frac{R_{s}}{2}<r<R_{s}$ could be a Cauchy horizon zone but this needs to be evaluated. In other words, for this SCK light geodesic metric, the energy is not directed towards the center at $r \rightarrow 0$ (singularity) but for an area around the edge near the $\mathrm{EH}$ (a thick skin $\frac{R_{s}}{2}$ or a ring of the $\mathrm{BH}$ rather than a membrane at $R_{s}$ ).

One can evaluate whether the total relativistic energy invariant is satisfied in this zone, $\frac{R_{s}}{2}<r<R_{s}$, either,

$$
\frac{E^{2}}{c^{2}}-p^{2}=m^{2} c^{2}
$$

with

$$
\begin{gathered}
E^{2}=\gamma^{2} m^{2} c^{4}=\frac{m^{2} c^{4}}{1-\frac{v^{2}}{c^{2}}} \\
p^{2}=\gamma^{2} m^{2} v^{2}=\frac{m^{2} v^{2}}{1-\frac{v^{2}}{c^{2}}} \\
v^{2}=c^{2}\left(\frac{1}{2}\left(1-\frac{R_{s}}{r}\right)+\frac{1}{2}\left(1-\frac{R_{s}}{r}\right)^{2}\right)=c^{2}\left(1-\frac{3 R_{s}}{2 r}+\frac{R_{S}^{2}}{2 r^{2}}\right)=c^{2} \Psi\left(r, R_{s}\right)
\end{gathered}
$$

Let's substitute the expression of $v^{2}$ in the invariant equation we find: 


$$
\begin{gathered}
E=\frac{m c^{2}}{\sqrt{1-\Psi}}, \quad p=\frac{\sqrt{\Psi} m c}{\sqrt{1-\Psi}} \\
\frac{E^{2}}{c^{2}}-p^{2}=\frac{m^{2} c^{2}}{1-\Psi}-\frac{m^{2} c^{2} \Psi}{1-\Psi}=m^{2} c^{2} \frac{1-\Psi}{1-\Psi}=m^{2} c^{2}
\end{gathered}
$$

Thus, we see that the total relativistic energy invariant is satisfied for the entire course of the photon including this particuliar zone. The result is remarkable since this quantum light geodesics properly satisfied the total relativistic energy, but the photons have imaginary speed in the $\mathrm{BH}$ zone and an imaginary momentum $p$. An imaginary momentum is not possible in quantum mechanics because it's a measurable quantity, but this $\mathrm{BH}$ zone is unconventional in terms of spacetime and in addition, we can not measure anything in this $\mathrm{BH}$ zone. Also, the Lorentz factor in this $\mathrm{BH}$ zone is less than $1(\gamma<1)$ which is strange because not conform to $E_{\min }=m c^{2}$. This corresponds to a proper time $\Delta \tau$ in this zone which is greater than $\Delta t$ outside this $\mathrm{BH}$ zone (inverse of normal case). This $\mathrm{BH}$ zone looks like a cavity where the energy is trapped, and the laws of physics seem partially different. However, electromagnetic energy is not necessarily in the conventional state.

The solution found corresponds in part to that found with the so-called EinsteinGauss-Bonnet (EGB) approach. Indeed, this approach makes it possible to find different solutions in the case of BH. In particular, [14] Glavan et al., using this EGB approach, show that a solution predicts the existence of 2 horizons $r_{ \pm}^{H}$ according to the value of a parameter $\alpha \quad(\alpha \geq 0)$ either.

$$
r_{ \pm}^{H}=\frac{R_{s}}{2}\left[1 \pm \sqrt{1-\frac{16 \pi \alpha}{G M^{2}}}\right]
$$

For $\alpha=0$, we find the SC case of the GR, either an EH at $R_{s}$. For $\alpha=\frac{G M^{2}}{16 \pi}$ we find an EH at $\frac{R_{s}}{2}$. Finally for $0<\alpha<\frac{G M^{2}}{16 \pi}$ we find 2 horizons and the appearance of a WH for $0<r<r_{-}^{H}$. However, we see that the horizons found $r_{ \pm}^{H}$ do not exactly match those found with this SCK.

With this SCK metric, it allows us to better understand the distribution of energy in the $\mathrm{BH}$ since this energy is not directed towards the singularity but towards a physical zone near the EH. We can estimate the energy density of such a $\mathrm{BH}$ in the area $\frac{R_{s}}{2}<r<R_{s}$, either.

$$
\rho=\frac{M}{V}=\frac{M}{\frac{4}{3} \pi\left(R_{s}^{3}-\left(\frac{R_{s}}{2}\right)^{3}\right)}=\frac{M}{\frac{28 \pi G^{3} M^{3}}{3 c^{6}}}=\frac{3 c^{6}}{28 \pi G^{3} M^{2}}
$$

Thus, the density decreases with the increase in mass $M$ as is the case of the conventional $\mathrm{SCH}$ and it is independent of the size. According to this metric, there is no limit to the growth of $\mathrm{BH}$ in terms of mass. Considering that the miminal radius of the $\mathrm{BH}$ is in order $2 l_{P}$ (inner $\mathrm{WH}$ and outer $\mathrm{BH}$ zone), we find that the maximum energy density of such a $\mathrm{WH}-\mathrm{BH}$ pair is: 


$$
\rho_{\text {max }}=\frac{3}{32 \pi} \rho_{\text {Planck }}
$$

In regards to the state of energy (matter) in the $\mathrm{BH}$ zone, it is possible to try to determine this state which corresponds in part to a gas in a cavity. For fermions, it can be thought that matter follows the Fermi-Dirac statistic at very low temperature would be in a degenerate state with minimum energy $\varepsilon_{0}$ occupied by fermions. The parameters $E_{F}, T_{F}, P, p, S$ for $N$ electrons, protons and neutrons in volume $V$ of gas using this statistic can be estimated, but the results would be purely speculative [15].

An important point is to be emphasized. Indeed, given this central area of the type $\mathrm{WH}$, the complete evaporation of the $\mathrm{BH}$ seems possible without leaving a singularity to $r=0$ because the energy does not get to the point $r=0$. Ideed, one can imagine that the last mass part of the $\mathrm{BH}$ is evaporated and the $\mathrm{WH}$ explodes at the end without any energy involved.

Figure 3 shows a schematic of the formation of a $\mathrm{BH}$ and $\mathrm{WH}$ with this quantum light geodesic. In $t_{0}^{\prime}$ the collapse begins. An EH is created at $t_{1}^{\prime}$ and the formation of a $\mathrm{BH}$ and $\mathrm{WH}$ begin. In $t_{2}^{\prime}$ the process is completed and the final mass of the $\mathrm{BH}$ produces a radius $R_{s}$ and a WH of radius $\frac{R_{s}}{2}$.

A more detailed simulation would allow us to better see the appearance of such a $\mathrm{BH}$. It is important to note that all parameters outside the $\mathrm{EH}$ are the same for the SCK light geodesic metric (spherical symmetry for $r>R_{s}$ ). In addition, SCK light geodesic metric predicts energy near the border of the $\mathrm{EH}$.

\section{Hawking Temperature and BH Evaporation by Tunnel Effect}

In 1975, Hawking proposed an ingenious mechanism of energy evaporation of $\mathrm{BH}$ that he attributes to a quantum effect of creating particles and antiparticles [16]. With this metric, energy (mass) remains near on the $\mathrm{EH}$, which allows us to imagine a process of energy evaporation of $\mathrm{BH}$ by the quantum tunnel effect [17] [18]. To illustrate the process, a semi-classical estimate of this effect can be made. If the mass is made up mainly of hadron (proton) and a quantum tunnel effect allows a tiny amount to pass beyond the $\mathrm{EH}$, we can estimate the energy emitted by the $\mathrm{BH}$ with the quantum tunnel effect by knowing the energy of the emitted particle as well as the number. Let's say an energy barrier inside the $\mathrm{EH}$ as $U$ of few diameters of the protons $=n r_{p}$. A first simplified model of quantum tunnel effect transmission through a rectangular barrier predicts a $T$ transmission factor [19].

$$
T=t^{2}=\frac{4 K^{2} k^{2}}{\left(K^{2}+k^{2}\right)^{2} \sinh ^{2}(K d)+4 K^{2} k^{2}}
$$

with: $K=2 \pi \sqrt{2 m_{p}\left(U-E_{p}\right)} / h$

$$
k=2 \pi \sqrt{2 m_{p} E_{p}} / h
$$




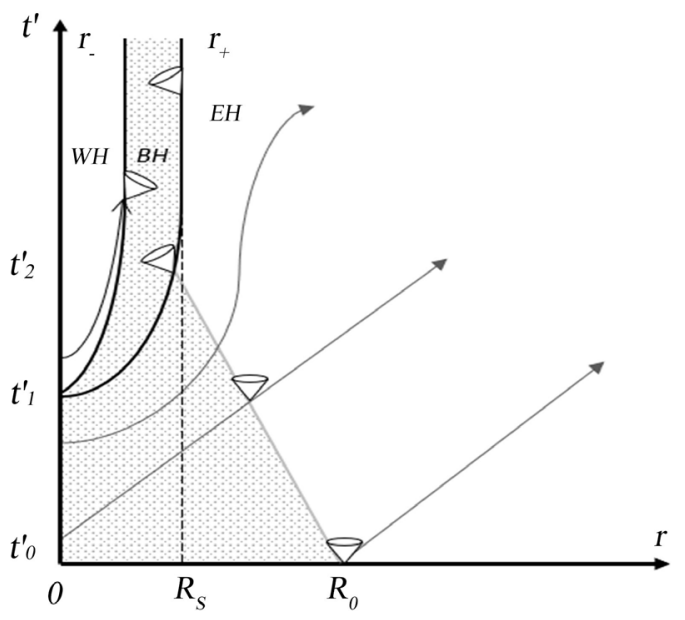

Figure 3. Schematic of the formation of a $\mathrm{BH}$ and WH with the collapse of a star with SCK.

In the case of the $\mathrm{BH}$ and this tunnel effect, the kinetic energy is that of the protons $E_{p}$ estimated by the internal energy of the proton at very low Hawking temperature. The barrier is the gravitational $U$ energy at the $\mathrm{EH}$ to release a particle $r \rightarrow \infty$ (proton). The value of the energy transmitted must be that of Hawking's radiation $\dot{E}_{H}$ at Hawking's temperature $T_{H}$ (evaporation). Let's evaluate the tunnel effect, the proton's energy.

$$
\begin{gathered}
E_{p}=\pi k_{B} T_{H} \\
U=\left|\sqrt{1-\frac{R_{s}}{r \rightarrow R_{s}}}-1\right| \frac{1}{\sqrt{1-v^{2} / c^{2}}} m_{p} c^{2} \sim m_{p} c^{2} \\
E_{H}=A \sigma T_{H}^{4}
\end{gathered}
$$

For example, we could estimate the parameters of the quantum tunnel effect for the $\mathrm{BH}$ of $\mathrm{M} 87$ assuming a static $\mathrm{BH}$, we find the following parameters:

$$
\begin{gathered}
M \sim 6.5 M_{\odot}=1.287 \times 10^{31} \mathrm{~kg} \\
R_{s}=1.9 \times 10^{13} \mathrm{~kg} \\
T_{H}=9.53 \times 10^{-18} \mathrm{~K} \\
\dot{E}_{H}=2.15 \times 10^{-48} \mathrm{~W} \\
E_{p}=4.13 \times 10^{-40} \mathrm{~J} \\
U \sim 1.50 \times 10^{-10} \mathrm{~J} \\
K=6.72 \times 10^{15} \mathrm{~m}^{-1} \\
k=1.11 \times 10^{1} \mathrm{~m}^{-1} \\
d \sim 6 r_{p} \mathrm{~m}
\end{gathered}
$$

We find the transmission factor.

$$
T=1.52 \times 10^{-58}
$$

We find that the probability of proton transmision over the EH is extremely 
low but the number of protons, near the $\mathrm{EH}$ is very high. Suppose the kinetic energy of the protons without the barrier $U$ i.e. without the $\mathrm{BH}$ and without the gravitation. Protons are free. The possibility of motion of protons can be done in both directions. 50\% $r_{-}$and $50 \% r_{+}$. Thus, the possible proton flow towards $r \rightarrow \infty$ (without $\mathrm{BH}$ ) is.

$$
\dot{m} \sim \frac{1}{2} \rho A v \sim \rho 2 \pi R_{s}^{2} v
$$

with $(v \ll c$, non relativistic)

$$
\begin{gathered}
v \sim \sqrt{\frac{2 E_{p}}{m_{p}}} \sim 7.03 \times 10^{-7} \mathrm{~m} \cdot \mathrm{s}^{-1} \\
\rho=\frac{3 c^{6}}{28 \pi G^{3} M^{2}} \sim 0.502 \mathrm{~kg} \cdot \mathrm{m}^{-3}
\end{gathered}
$$

We find,

$$
\dot{m} \sim 8.11 \times 10^{20} \mathrm{~kg} \cdot \mathrm{s}^{-1}
$$

However, considering a tunnel effect with a barrier $U\left(d=6 r_{p}\right)$ we find this fraction of transmitted proton.

$$
\dot{N}_{p T}=\frac{T \dot{m}}{m_{p}}=7.37 \times 10^{-11} \mathrm{~s}^{-1}
$$

Finally, the energy emitted by tunnel effect to temperature $T_{H}$ expresses itself as.

$$
\dot{E}_{p T}=\dot{N}_{p T} E_{p}=7.37 \times 10^{-11} \mathrm{~s}^{-1} \times 4.13 \times 10^{-40} \mathrm{~J}=3.04 \times 10^{-50} \mathrm{~W}
$$

If we compare it with Hawking's radiation, we find.

$$
\frac{\dot{E}_{p T}}{\dot{E}_{H}}=\frac{3.04 \times 10^{-50} \mathrm{~W}}{2.15 \times 10^{-48} \mathrm{~W}} \sim 0.014
$$

The value found is relatively well predicted given the uncertainty surrounding the tunnel effect (type, particle, barriers, others). The ratio can be changed by changing the thickness of the barrier $d$. The value found changes rapidly with the thickness of the barrier. With this tunnel effect, the BH of M87 will be evaporated for a time.

$$
t_{\text {eva }}=\frac{M}{T \dot{m}} \sim \frac{1.287 \times 10^{31} \mathrm{~kg}}{1.23 \times 10^{-37} \mathrm{~kg} \cdot \mathrm{s}^{-1}} \sim 10^{77} \mathrm{~s} \sim 10^{60} \mathrm{~Gy}
$$

Figure 4 shows the effects of the thickness of the barrier $d$ in terms of the radius of the proton on the ratio of energy evporated $\frac{\dot{E}_{p T}}{\dot{E}_{H}}$ ratio for a variation in the $\mathrm{BH}$ mass from $6.5 M_{\odot}$ to $6.5 \times 10^{9} M_{\odot}$. We see that, despite the great variation in the mass of the $\mathrm{BH}$, the size of the quantum barrier varies very little for the desired ratio of 1 . However, this example of tunnel effect predicts a free proton transfer to the other side of the $\mathrm{EH}$, which is not necessarily black body radiation as predicted by Hawking's theory (electromagnetic). Finally, the value $d \sim 6 r_{p}$ that yields the ratio close to 1 does not have a particular justification. 


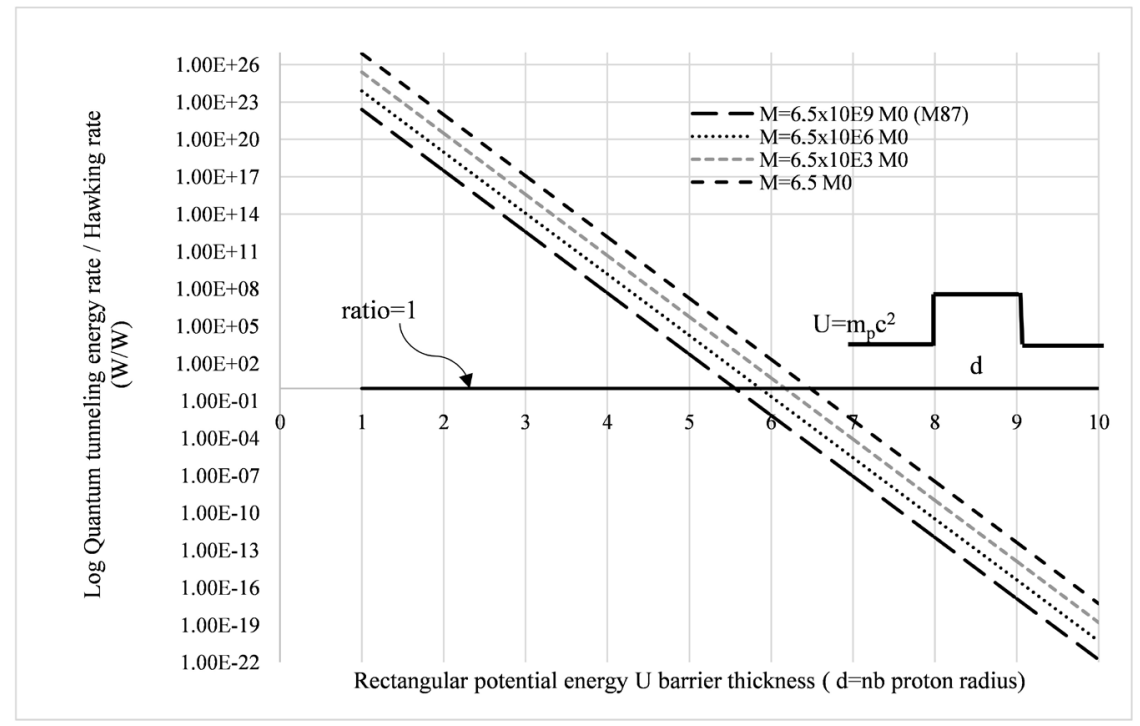

Figure 4. Ratio of proton quantum tunneling effect to Hawking's radiation function of quantum barrier thickness $d$.

\section{Gravitational Wave and Validation of This Quantum Light Geodesic Metric}

The detection of GW150914 gravitational waves in 2016 [20] has given rise to several explanations and simulations of the collision of $\mathrm{BH}$ with inspiraling process according to a numerical approach called post-Newtonian [21]. One approach is to consider the masses as a point source [22]. A mass, called chirp mass $\mathrm{M}$, had been calculated which allows to estimate the masses of the two bodies in mutual $m_{1}$ et $m_{2}$ rotation. This SCK light geodesic metric predicts that the energy of the $\mathrm{BH}$ is located around the edge of the $\mathrm{EH}$, which is a major difference that does not allow the masses to be considered as point sources when the collision occurs. This energy distribution around rotating $\mathrm{BH}$ could be a special signature to validate this metric when observing gravitational waves from the fusion of $2 \mathrm{BH}$. Indeed, if the received signal takes a particular form (amplitude or frequency deformation longer duration) when the separation of the $\mathrm{BH}$ will be that of the $\sim R_{s}$ largest, then it could be an indication that the energy is well around and the fusion leads to a reorganization of the energy around the new EH of the new BH formed. The observed and filtered signal clearly distinguishes a state of reorganization when the separation radius decreases beyond what could confirm the presence of energy in the zone of 1 to $1.5 R_{s}$ (see Figure 2 of Abbott et al., 2016 [20]). Indeed, for a central energy concentration, the measured signal should show a continuous increase up to say near $r \rightarrow 0$ while we see a decrease towards $r \rightarrow 1 \sim 1.5 R_{s}$. The future will certainly allow us to better understand the merging effects of $\mathrm{BH}$ and to determine more precisely the internal nature of $\mathrm{BH}$.

\section{Conclusion}

In this article we hypothesized that the arrow of time and space evolve in a dis- 
continuous way in the form of quanta $\left(t_{P}, l_{P}\right)$. We applied this reasoning to the light geodesics of Schwarzschild's metric and obtained different characteristics of the $\mathrm{BH}$ obtained. Indeed, quantum light geodesics show that inside the $\mathrm{BH}$ a $\mathrm{WH}$ is formed, and the mass (energy) is not directing towards the singularity $r \rightarrow 0$ but rather around the $\mathrm{BH}$ near the $\mathrm{EH}$ as a thick skin. Subsequently, as mass (energy) is found directly at the EH, we applied the tunnel effect in simple semi-classical analysis, and we obtained that particles, like protons, can leave the $\mathrm{EH}$ and that the energy associated with them is in the order of magnitude of Hawking's radiation. However, the energy of the protons is not necessarily identified with that of the black body (electromagnetic). Finally, it would be interesting to see the impacts of this quantum light geodesics $\left(\mathrm{d} s=l_{P}\right)$ on other cases like Kerr's metric.

\section{Funding Statement}

Funding for this article was supported by the University of Quebec at Chicouti$\mathrm{mi}$.

\section{Acknowledgements}

The author would like to thank the members of his family, especially his spouse (Danielle) as well his childrens (Pierre-Luc, Vincent, Claudia). Finally, thanks to the University of Quebec at Chicoutimi and to the colleagues of the Department of Applied Sciences for their supports in the realization of this work.

\section{Conflicts of Interest}

The author declares no conflicts of interest regarding the publication of this paper.

\section{References}

[1] Perron, J. (2021) An Alternative to Dark Matter? Part 1: The Early Universe $\left(t_{p}\right.$ to $10^{-9} \mathrm{~s}$ ), Energy Creation the Alphaton, Baryogenesis. Journal of High Energy Physics, Gravitation and Cosmology, 7, 784-807. https://doi.org/10.4236/jhepgc.2021.73046

[2] Akiyama, K., Alberdi, A., et al. (2019) First M87 Event Horizon Telescope Results. I. The Shadow of the Supermassive Black Hole. The Astrophysical Journal Letters, 875, Article No. L1.

[3] Schwarzschild, K. (1916) A propos du champ gravitationnel d'une boule faite de liquide incompressible selon la théorie d'Einstein, Rapports de réunion de l'Académie royale des sciences de Prusse à Berlin. Ed. Académie Allemande des Sciences, Berlin, 424-434.

[4] Kerr R.P. (1963) Gravitational Field of a Spinning Mass as an Example of Agebraically Special Metrics. Physical Review Letters, 11, 237-238. https://doi.org/10.1103/PhysRevLett.11.237

[5] Newman E.T., Couch, E., Chinnapared, K., Exton, A., Prakash, A. and Torrence, R. (2004) Metric of a Rotating, Charged Mass. Journal of Mathematical Physics, 6, 918 919. https://doi.org/10.1063/1.1704351 
[6] Bardoux, Y. (2012) Trous noirs dans des théories modifiés de la gravitation. Thèse, Université Paris Sud, Paris.

[7] Dewitt, B. and Seligman, D.B. (2003) The Global Approach to Quantum Field Theory. Oxford University Press, Oxford.

[8] Rovelli, C. (2008) Loop Quantum Gravity. Living Reviews in Relativity, 11, Article No. 5. https://doi.org/10.12942/lrr-2008-5

[9] McGrew, W.F., Zhang, X., Fasano, R.J., et al. (2018) Atomic Clock Performance Enabling Geodesy Below the Centimetre Level. Nature, 564, 87-90.

https://doi.org/10.1038/s41586-018-0738-2

[10] Kazakov, D.I. and Solodukhin, S.N. (1993) On Quantum Deformation of the Schwarzschild Solution. Nuclear Physics B, 429, 153-176.

[11] Nozari, K., Hajebrahimi, M. and Saghafi, S. (2020) Quantum Corrections to the Accretion onto a Schwarzschild Black Hole in the Background of Quintessence. The European Physical Journal C, 80, Article No. 1208. https://doi.org/10.1140/epjc/s10052-020-08782-2

[12] Zeldovich Y., Novikov, I.D. and Starobinski, A.A. (1974) Quantum Effects in White Holes. Soviet Physics. Journal of Experimental and Theoretical Physics, 39, 933-939.

[13] Narlikar, J.V. and Apparao, K.M.V. (1975) White Hole and High Energy Astrophysics. Astrophysics and Space Science, 35, 321-336.

https://doi.org/10.1007/BF00637001

[14] Glavan, D. and Lin, C. (2020) Einstein-Gauss-Bonnet Gravity in 4-Dimensional Space-Time. Physical Review Letters, 124, Article ID: 081301.

https://doi.org/10.1103/PhysRevLett.124.081301

[15] Landau, L.D. and Lifshitz, E.M. (1969) Statistical Physics. Pergamon Press, Oxford, $144 \mathrm{p}$.

[16] Hawking, S.W. (1975) Particle Creation by Black Holes. Communications in Mathematical Physics, 43, 199-220. https://doi.org/10.1007/BF02345020

[17] Chatterjee. B., Ghosh, A. and Mitra, P. (2008) Tunnelling from Black Holes and Tunnelling into White Holes. Physics Letters B, 661, 307-311. https://doi.org/10.1016/j.physletb.2008.02.034

[18] Parikh, K.P. and Wilczek, F. (2000) Hawking Radiation as Tunneling. Physical Review Letters, 85, 5042-5045. https://doi.org/10.1103/PhysRevLett.85.5042

[19] Cohen-Tannoudji, C. and Laloe, F. (2018) Mécanique Quantique. Tome 1, EDP Sciences, Oxford.

[20] Abbott, B.P., et al, (2016) Observation of Gravitational Waves from a Binary Black Hole Merger. Physical Review Letters, 116, Article ID: 061102.

[21] Blanchet, L. and Lyer, B.R. (2003) Third Post-Newtonian Dynamics of Compact Binaries: Equations of Motion in the Centre-Of-Mass Frame. Classical and Quantum Gravity, 20, 755-776. https://doi.org/10.1088/0264-9381/20/4/309

[22] Cutler, C. and Flanagan, E.E. (1994) Gravitational Waves from Merging Compact Binaries: How Accurately Can One Extract the Binary's Parameters from the Inspiral Waveform? Physical Review D, 49, 2658-2697. https://doi.org/10.1103/PhysRevD.49.2658 